\section{Prostatakarzinom: Krankheitsspezifische Mortalität sinkt}

Immer weniger Patienten, bei denen heute ein Prostatakrebs diagnostiziert wird, versterben an der Krankheit. Viel häufiger bringen auch diese Patienten etwa die üblichen Folgen mangelnder Fitness und falscher Ernährung oder andere Tumoren ins Grab. Deshalb empfehlen US-amerikanische Autoren, im Rahmen einer Prostatakrebsbehandlung die Chance zu nutzen, auch Risikofaktoren für andere Krankheiten anzugehen.

D as PSA-Screening hat vieles verändert. Wie die amerikanische SEERStudie („surveillance, epidemiology and results") gezeigt hat, war bei $81 \%$ der Männer, bei denen zwischen 2001 und 2007 ein Prostatakrebs diagnostiziert wurde, der Tumor noch lokal begrenzt. So ist der Krebs in vielen Fällen nicht mehr die Todesursache. Woran Schweden und Amerikaner mit Prostatakrebs letztlich versterben, untersuchten US-amerikanische Wissenschaftler anhand populationsbasierter Daten des Schwedischen Krebsregisters von 1961 bis 2008 sowie des amerikanischen SEER-Programms von 1973 bis 2008. Insgesamt wurden die Daten von über 600.000 Prostatakrebspatienten ausgewertet.

Bei etwa jedem zweiten Schweden war das Prostatakarzinom nach Einführung des PSA-Screenings diagnostiziert worden. Insgesamt waren 143.189 Männer im Beobachtungszeitraum verstorben. $35 \%$ der Patienten erlagen während des Follow-up ihrer Prostatakrebserkrankung. Beim Vergleich der Todesursachen fielen $52 \%$ auf den Prostatakrebs, $17 \%$ auf die ischämische Herzkrankheit und $6 \%$ auf andere Krebserkrankungen. In den USA war der Prostatakrebs bei zwei Dritteln der Patienten in Zeiten des PSA-
Screenings diagnostiziert worden. 259.021 Patienten starben im Beobachtungszeitraum. Allerdings erlagen nur $16 \%$ aller Patienten, bei denen ein Prostatakrebs diagnostiziert worden war, tatsächlich ihrer Erkrankung. Mit 30\% war der Prostatakrebs immer noch häufigste Todesursache, dicht darauf folgte aber mit $24 \%$ die ischämische Herzkrankheit. $15 \%$ der Prostatakrebspatienten starben letztlich an anderen Krebserkrankungen.

Deutlichen Einfluss auf die spezifischen Mortalitätsraten hatte offenbar das PSAScreening. Nach seiner Einführung wurden Prostatakarzinome ab 1994 viel häufiger und viel früher diagnostiziert, allerdings auch überdiagnostiziert. Nach den statistischen Berechnungen starben in Schweden 39\% der Männer innerhalb von fünf Jahren einen krankheitsspezifischen Tod, wenn die Diagnose vor dem PSAScreening gestellt wurde, und nur $14 \%$ bei späterer Diagnose. In den USA lagen die spezifischen Sterberaten entsprechend bei $23 \%$ oder $6 \%$.

Die kumulative Fünf-Jahres-Inzidenz für einen krankheitsspezifischen Tod infolge Prostatakarzinom ist in den USA in den vergangenen Jahrzehnten deutlich gesunken. Insgesamt errechneten die $\mathrm{Au}$ - toren eine Fünf-Jahres-Sterberate von $29 \%$ für die Schweden und $11 \%$ für die Amerikaner. Während von den amerikanischen Männern, deren Prostatakrebs in den 1970er-Jahren diagnostiziert worden war, noch etwa $24 \%$ die kommenden fünf Jahre nicht überlebten, waren dies bei einer Diagnosestellung in den 1990ern nur noch $8 \%$. Die Rate für die ischämische Herzkrankheit als Todesursache bei dieser Patientengruppe reduzierte sich in diesem Zeitraum von $12 \%$ auf $6 \%$. Bei den Schweden ist die kumulative Fünf-Jahres-Inzidenz für einen Tod infolge Prostatakarzinom von $41 \%$ bei Diagnosestellung in den 1960er-Jahren auf $25 \%$ gesunken, wenn die Krankheit in den 1990er-Jahren entdeckt wurde. Die Fünf-Jahres-Inzidenz für die ischämische Herzkrankheit als Todesursache der Schweden hat sich vergleichsweise wenig verändert.

Fazit: Sowohl in Schweden als auch in den USA ist das Risiko, an einem Prostatakarzinom zu versterben, in den letzten Jahrzehnten deutlich zurückgegangen. Dass die krankheitsspezifische Sterberate in Schweden immer noch höher liegt als in den USA, könnte möglicherweise einem konsequenteren und früheren PSA-Screening und entsprechend kurativer Behandlung in den USA zuzuschreiben sein. Lebensstilveränderungen könnten das Risiko verringern, an ischämischer Herzkrankheit und möglicherweise auch anderen Krebserkrankungen zu versterben, meinen die Autoren. Dr. Christine Starostzik

Epstein MM et al. Temporal Trends in Cause of Death Among Swedish and US Men with Prostate Cancer. J Natl Cancer Inst 2012; 104: 1335-42 